\title{
Publisher Correction: Complete reconstitution of the diverse pathways of gentamicin B biosynthesis
}

Yeon Hee Ban, Myoung Chong Song (D), Jae-yeon Hwang, Hea-lyung Shin, Hak Joong Kim (D), Seung Kon Hong (DD, Na Joon Lee, Je Won Park, Sun-Shin Cha, Hung-wen Liu (D) and Yeo Joon Yoon (D)

Correction to Nature Chemical Biology https://doi.org/10.1038/s41589-018-0203-4, published online 14 January 2019

In the version of this article originally published, the name of co-author Hea-lyung Shin was omitted from the text of the equal contribution statement. The statement should read "These authors contributed equally: Yeon Hee Ban, Myoung Chong Song, Jae-yeon Hwang, Hea-lyung Shin, Hak Joong Kim, Seung Kon Hong”. The error has been corrected in the HTML and PDF versions of the paper.

Published online: 2 June 2020

https://doi.org/10.1038/s41589-020-0577-y

(c) The Author(s), under exclusive licence to Springer Nature America, Inc. 2020 